\title{
Análise imuno-histoquímica de Ki-67 e $\alpha-S M A$ em ceratocisto odontogênico, ameloblastoma e folículo pericoronário
}

\author{
Immunohistochemical analysis of Ki-67 and a-SMA in odontogenic keratocyst, \\ ameloblastoma and pericoronal follicle
}

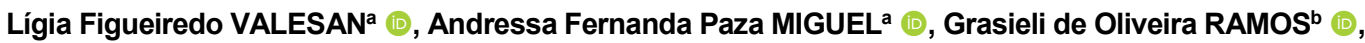 \\ Elena Riet Correa RIVERO ${ }^{a}$ (D) , Kamile Leonardi DUTRA-HORSTMANN ${ }^{\text {a* }}$ (1) \\ aUFSC - Universidade Federal de Santa Catarina, Florianópolis, SC, Brasil \\ bUNOESC - Universidade do Oeste de Santa Catarina, Joaçaba, SC, Brasil
}

\begin{abstract}
Como citar: Valesan LF, Miguel AFP, Ramos GO, Rivero ERC, Dutra-Horstmann KL. Análise imuno-histoquímica de Ki-67 e $\alpha$-SMA em ceratocisto odontogênico, ameloblastoma e folículo pericoronário. Rev Odontol UNESP. 2019;48:e20190067.

https://doi.org/10.1590/1807-2577.06719
\end{abstract}

\begin{abstract}
Resumo
Introdução: Os ameloblastomas (AM) são considerados os tumores odontogênicos mais comuns da cavidade bucal, apresentando grande importância clínica devido à sua agressividade, capacidade infiltrativa e comportamento recorrente. De maneira semelhante, o ceratocisto odontogênico (CO) desperta a atenção por ter um comportamento agressivo e altas taxas de recorrência em relação aos outros cistos de desenvolvimento. Objetivo: Avaliar e comparar o índice de proliferação epitelial e a presença de miofibroblastos em CO e AM, por meio dos anticorpos Ki-67 e $\alpha$-SMA, respectivamente. Metodologia: Foram selecionados 15 casos de AM e 24 casos de CO para investigação imuno-histoquímica das proteínas Ki-67 e $\alpha$-SMA. Um grupo de sete folículos pericoronários (FP) foi incluído como controle de tecido odontogênico normal. A média de células positivas foi calculada para cada marcador. Resultado: 0 teste de Kruskal-Wallis revelou que a expressão de ambos os marcadores foi maior nos casos de CO, quando comparada à expressão em AM e FP. Segundo o teste de Mann-Whitney, a expressão dos marcadores foi semelhante entre os subtipos de AM. Conclusão: A alta expressão de Ki-67 e $\alpha$-SMA observada em CO poderia estar associada ao comportamento agressivo desta lesão em relação aos outros cistos de desenvolvimento. Por outro lado, a expressão semelhante destas proteínas nos casos de AM e FP, assim como nos subtipos de AM, poderia indicar que outros fatores, além do potencial proliferativo, estariam associados ao comportamento clínico agressivo do AM.
\end{abstract}

Descritores: Ameloblastoma; cistos odontogênicos; antígeno Ki-67; miofibroblastos.

\begin{abstract}
Introduction: Ameloblastomas (AM) are considered the most common odontogenic tumors, with great clinical importance linked to its locally aggressive, infiltrative, and recurrent behavior. The odontogenic keratocyst $(\mathrm{OK})$ represents a peculiar cyst that also draws attention because of its aggressive behavior and high rates of local recurrence. Objective: The aim of this study was to evaluate the cell proliferation index and the presence of myofibroblasts in AM and OK using Ki-67 and $\alpha$-SMA antibodies, respectively. Methodology: Fifteen cases of AM and 24 cases of OK were selected for immunohistochemical investigation of the proteins $\alpha$ SMA and Ki-67. A group of 7 pericoronal follicles (PF) was included as control for normal odontogenic tissue. The mean percentage of positive cells was calculated for each protein. Result: Kruskal-Wallis test for both markers showed that expression was higher in OK, when compared to AM and PF. Mann-Whitney test showed no statistical difference when comparing the expression of both markers between the subtypes of AM. Conclusion: Elevated expression of Ki-67 and $\alpha$-SMA in OK may be associated with the aggressive clinical behavior of this cyst in comparison to other development cysts. The similar expression of the markers observed among AM and PF cases, as well as among subtypes of AM, may indicate that other factors, besides proliferative potential, are associated with the aggressive behavior of AM.
\end{abstract}

Descriptors: Ameloblastoma; odontogenic cysts; antigen Ki-67; myofibroblast. 


\section{INTRODUÇÃO}

Em 2005, na terceira edição da Classificação Mundial de Tumores de Cabeça e Pescoço da Organização da Mundial da Saúde (OMS), o Ceratocisto Odontogênico (CO) foi classificado como tumor e renomeado Tumor Odontogênico Ceratocístico (TOC). A mudança baseou-se no comportamento clínico da lesão, na sua elevada taxa de recidiva e, principalmente, na presença da mutação do gene $P T C H^{1}$. No entanto, em janeiro de 2017, o TOC foi reclassificado como cisto e renomeado para $\mathrm{CO}$, devido à falta de evidências que suportassem mantê-lo na categoria de tumor² . O CO é um cisto com potencial de crescimento anteroposterior, com taxa de recorrência em torno de $21 \%$ e tendência à destruição de estruturas, especialmente se associado à síndrome do carcinoma basocelular nevóide ${ }^{3,4}$. Histologicamente, o CO apresenta elevada expressão imuno-histoquímica de proteínas relacionadas a proliferação e sobrevivência celular, angiogênese e remodelação óssea, sendo que alguns desses marcadores foram associados ao comportamento mais agressivo desta lesão em relação aos outros cistos 4 .

A terminologia dos ameloblastomas também apresentou mudanças na nova Classificação Mundial de Tumores de Cabeça e Pescoço, tornando-se mais simplificada e restringindo-se a Ameloblastoma (AM), Ameloblastoma Unicístico (AU) e Ameloblastoma Extraósseo/Periférico $(\mathrm{AE})^{2}$. 0 termo "sólido/multicístico", antes usado para o ameloblastoma convencional, foi removido por não possuir significância biológica e por gerar confusão com o subtipo AU2. Dentre os ameloblastomas, o AM é o subtipo mais frequente e também o que apresenta comportamento clínico mais agressivo, com crescimento localmente infiltrativo ${ }^{2,5}$. A ressecção do tumor geralmente requer uma margem de segurança devido ao alto potencial de recorrência após curetagem simples, ressecção sem margem de segurança ou combinação de curetagem e crioterapia ${ }^{6}$. Por outro lado, o AU assemelha-se radiográfica e clinicamente a uma lesão cística, possibilitando abordagens de tratamento mais conservadoras com menores índices de recidiva ${ }^{2}$.

Histologicamente, o AM apresenta uma grande variação de padrões, sendo que os mais comuns são o tipo folicular e o plexiforme ${ }^{5}$. Por outro lado, o AU apresenta três variantes histopatológicas: luminal, intraluminal e mural, sendo este último o que apresenta pior prognóstico ${ }^{2}$. A caracterização da expressão gênica dos ameloblastomas mostrou uma elevada expressão de proteínas relacionadas à proliferação celular e remodelação óssea e tecidual, as quais poderiam estar relacionadas ao desenvolvimento e à progressão destas lesões ${ }^{7}$.

$\mathrm{Na}$ identificação da atividade celular proliferativa, o anticorpo monoclonal Ki-67 é um marcador biológico considerado confiável na avaliação da quantidade de células em proliferação e, além disso, o seu uso é simplificado e rápido ${ }^{8}$. Nos ameloblastomas, o aumento do índice de proliferação celular está associado positivamente ao risco de recorrência ${ }^{9}$, bem como a alta expressão de Ki-67 nas camadas celulares suprabasais do revestimento epitelial em CO poderia explicar o seu comportamento clinicamente agressivo ${ }^{10}$.

Outro indicador confiável de progressão tumoral é a expressão da proteína alfa-actina de músculo liso ( $\alpha$-SMA), que permite avaliar a presença de miofibroblastos (MFs). Os MFs são fibroblastos que possuem características semelhantes às de músculo liso, com capacidade contrátil devida à elevada expressão de $\alpha$-SMA ${ }^{11}$. Os MFs, além de produzirem colágeno, têm o potencial de facilitar a progressão de lesões epiteliais neoplásicas, podendo também favorecer o crescimento de cistos e tumores odontogênicos ${ }^{12}$.

Com o exposto, este estudo teve como objetivo avaliar o índice de proliferação celular e a presença de MFs utilizando os anticorpos Ki-67 e $\alpha$-SMA, respectivamente, com o intuito de obter uma melhor compreensão do comportamento biológico dos casos de CO, AM e AU. 


\section{METODOLOGIA}

\section{Seleção da Amostra}

Este estudo foi aprovado pelo Comitê de Ética em Pesquisa com Seres Humanos (CEPSH) da Universidade Federal de Santa Catarina, sob os números: 1.285.803 Parecer Plataforma Brasil / Certificado de Apreciação Ética (CAAE): 49115915.5.0000.0121.67.

Amostras e laudos histopatológicos foram selecionados de dois laboratórios de diagnóstico da universidade a que pertencem os autores. Foram selecionados 15 casos de ameloblastomas $(\mathrm{AU}=7 ; \mathrm{AM}=8)$ e 24 casos de CO. Como grupo controle de lesão não tumoral/cística, foram incluídos sete casos de FP de terceiros molares completamente inclusos, comprovados radiograficamente, e livres de processo inflamatório.

\section{Técnica Imuno-histoquímica}

As amostras foram submetidas à técnica de imuno-histoquímica pelo método da estreptavidina-biotina-peroxidase ${ }^{13,14}$. Foram obtidos cortes de $3 \mu \mathrm{m}$ de espessura das amostras fixadas em formol e emblocadas em parafina, os quais foram colocados em lâminas de vidro silanizadas com 3-aminopropiltrietoxissilano (Sigma-Aldrich, St. Louis, Missouri, USA). Foi realizada a desparafinização e a reidratação dos cortes, seguidas de imersão em solução de $\mathrm{H}_{2} \mathrm{O}_{2} /$ metanol $2 \%$ para o bloqueio da peroxidase endógena. A recuperação antigênica foi realizada em banho-maria por 45 min com uma solução tampão de citrato $0,01 \mathrm{M}(\mathrm{pH} 6,0)$, a $96^{\circ} \mathrm{C}$ (Merck, Darmstadt, Hessen, Alemanha). As amostras foram incubadas com os anticorpos primários Ki-67 e $\alpha$-SMA (MFs) durante a noite (Tabela 1). 0 Kit LSAB (Dako Corporation, Carpinteria, USA) foi utilizado para aplicação do anticorpo secundário biotinilado, seguido da incubação com o complexo estreptavidina-peroxidase. 0 cromógeno diaminobenzidina (DAB) (Biocare, Concord, CA, EUA) foi utilizado para a revelação da reação e a lâminas foram contrastadas com a Hematoxilina de Harris e montadas com Entellan (Merck, Alemanha). Após cada etapa, as lâminas foram lavadas em solução salina tamponada com fosfato (PBS) por cinco minutos. Foram incluídos controles positivos para todos os anticorpos (Tabela 1). 0 controle negativo de todas as reações foi realizado pela omissão do anticorpo primário.

Tabela 1. Detalhes dos anticorpos utilizados e seus respectivos controles positivos

\begin{tabular}{ccccc}
\hline Anticorpo & Clone & Fonte & Diluição & Controle positivo \\
\hline$\alpha$-SMA & Policlonal & Sigma & $1: 1000$ & $\begin{array}{c}\text { Vasos sanguíneos } \\
\text { Carcinoma } \\
\text { Ki-67 }\end{array}$ \\
Monoclonal - P6 & Biocare & $1: 100$ & epidermoide \\
\hline
\end{tabular}

\section{Análise Imuno-histoquímica}

O software NIH Image J® 1.45q (National Institutes of Health, Maryland, EUA) foi utilizado para analisar as imagens capturadas com uma câmera fotográfica (Cannon, A620, Beijing, China) acoplada ao microscópio de luz (Axiostar Plus, Carl Zeiss, Oberkochen, Alemanha), com magnitude de $400 \times$.

A presença de MFs foi analisada pela expressão de $\alpha$-SMA nos fibroblastos presentes no tecido conjuntivo logo abaixo do revestimento epitelial. 0 número total de células positivas e negativas foi contado em 10 campos consecutivos para cada caso. Células com coloração citoplasmática 
marrom foram consideradas positivas, excluindo-se as células inflamatórias, células endoteliais e pericitos, pois essas células podem interferir nos resultados devido à expressão de $\alpha$-SMA.

A imunopositividade para Ki-67 foi caracterizada pela coloração castanha do núcleo das células epiteliais. Para CO, as camadas basal e suprabasal foram analisadas; para o AM, as células periféricas das ilhas epiteliais foram avaliadas, e para o $\mathrm{AU}$, o revestimento do epitélio ameloblástico foi considerado. Para cada caso, as células epiteliais positivas e negativas foram contadas em 10 campos consecutivos. Para ambos os anticorpos, calculou-se a percentagem média de células positivas para cada caso. A concordância intraexaminador foi realizada antes da coleta dos dados oficiais para ambos os anticorpos. Os valores de kappa variaram de 0,89 a 0,94.

\section{Análise Estatística}

O teste estatístico de Kruskal-Wallis foi aplicado para a comparação da expressão do Ki-67 e $\alpha$-SMA entre os casos de ameloblastomas (AM+AU), CO e FP. O teste de Mann-Whitney foi utilizado para comparar os resultados entre AM e AU. A correlação entre Ki-67 e $\alpha$-SMA foi avaliada por meio do teste de correlação de Spearman. Os resultados foram expressos como média da porcentagem de células coradas \pm desvio padrão para ambos os marcadores (Tabela 2). Considerou-se o valor de $\mathrm{p} \leq 0,05$ para dados com significância estatística.

Tabela 2. Média percentual de células positivas \pm desvio padrão dos marcadores imuno-histoquímicos para Ki-67 e a-SMA em Ameloblastomas (AM+AU), AM, AU, CO e FP

\begin{tabular}{|c|c|c|c|c|}
\hline & \multicolumn{2}{|c|}{ Ki-67 } & \multicolumn{2}{|c|}{$\alpha$-SMA } \\
\hline & Média $( \pm D V)$ & $\mathbf{P}$ & Média $( \pm D V)$ & $\mathbf{P}$ \\
\hline AM & $6,93 \pm 2,3$ & 0.381 & $8,25 \pm 1,6$ & 0.817 \\
\hline AU & $8,94 \pm 1,6$ & & $7,71 \pm 1,1$ & \\
\hline Ameloblastomas $(\mathrm{AM}+\mathrm{AU})$ & $13,00 \pm 8,7 \mathrm{a}$ & & $15,83 \pm 6,5$ a & \\
\hline co & $31,81 \pm 6,9 \mathrm{~b}$ & $0.000^{*}$ & $28,80 \pm 8,3 b$ & $0.005^{*}$ \\
\hline FP & $12,75 \pm 5,8 \mathrm{a}$ & & $17,00 \pm 5,3 \mathrm{a}$ & \\
\hline
\end{tabular}

$\mathrm{AM}=$ ameloblastoma; $\mathrm{AU}=$ ameloblastoma unicístico; $\mathrm{CO}=$ ceratocisto odontogênico; $\mathrm{FP}=$ folículo pericoronário; $\mathrm{p}=$ valor de $\mathrm{p}$. aDiferenças entre AM e CO com $\mathrm{P}<0,05$. Kruskal-Wallis teste estatístico $(\mathrm{P}<0,05)$ com teste post hoc de Dunn-Bonferroni para comparações múltiplas. bDiferenças entre FP e CO com $\mathrm{P}<0,05$. Kruskal-Wallis teste estatístico $(\mathrm{P}<0,05)$ com teste post hoc de Dunn-Bonferroni para comparações múltiplas. Valores foram expressos como média \pm desvio padrão (\%). *valor estatisticamente significante.

\section{RESULTADO}

As expressões de Ki-67 e $\alpha$-SMA estão representadas nas Figuras 1 e 2 , respectivamente. Foi observado que a expressão de $\alpha$-SMA mostrou-se difusa no tecido conjuntivo (Figura 2). Por outro lado, a expressão nuclear de Ki-67 foi observada nas camadas basal e suprabasal do epitélio cístico de CO (Figura 1A) e por todo o epitélio ameloblástico em AU (Figura 1C), bem como nas células da periferia das ilhas tumorais de AM (Figura 2D). 

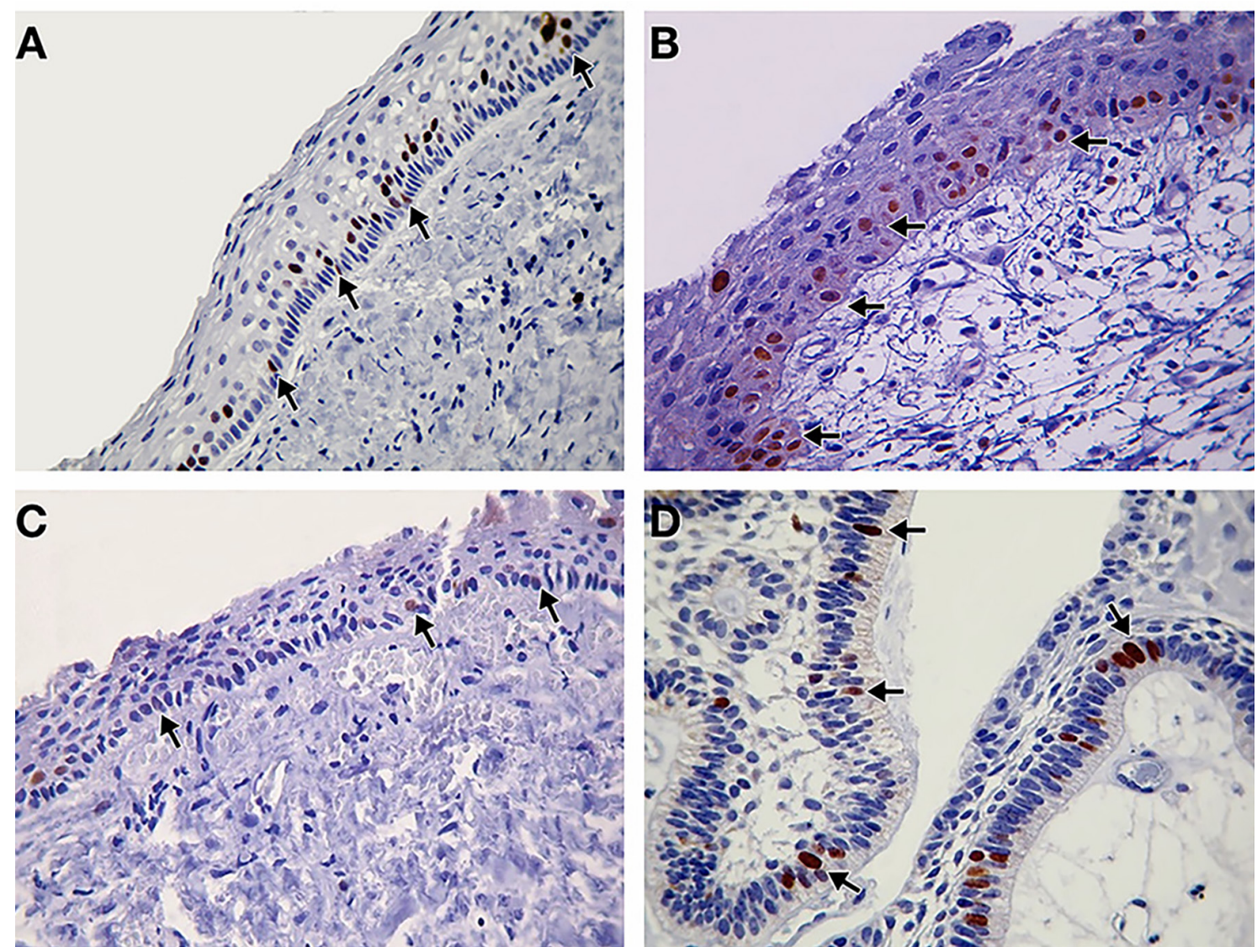

Figura 1. Fotomicrografia da expressão de Ki-67 no epitélio caracterizada pela marcação nuclear de cor marrom (seta) (LSAB, ×400): (A) Ceratocisto odontogênico exibindo epitélio pavimentoso estratificado com

camada basal em paliçada e paraceratina corrugada na superfície, revestindo a cápsula de tecido conjuntivo; (B) Folículo Pericoronário com epitélio odontogênico suportado por tecido conjuntivo fibroso e ausência de inflamação; (C) Ameloblastoma unicístico com epitélio tipicamente amoloblástico revestindo a cápsula cística; (D) Ameloblastoma com ilhas de epitélio odontogênico constituídas por células centrais frouxamente arranjadas semelhantes ao retículo estrelado do órgão do esmalte e uma camada periférica de células colunares altas. 

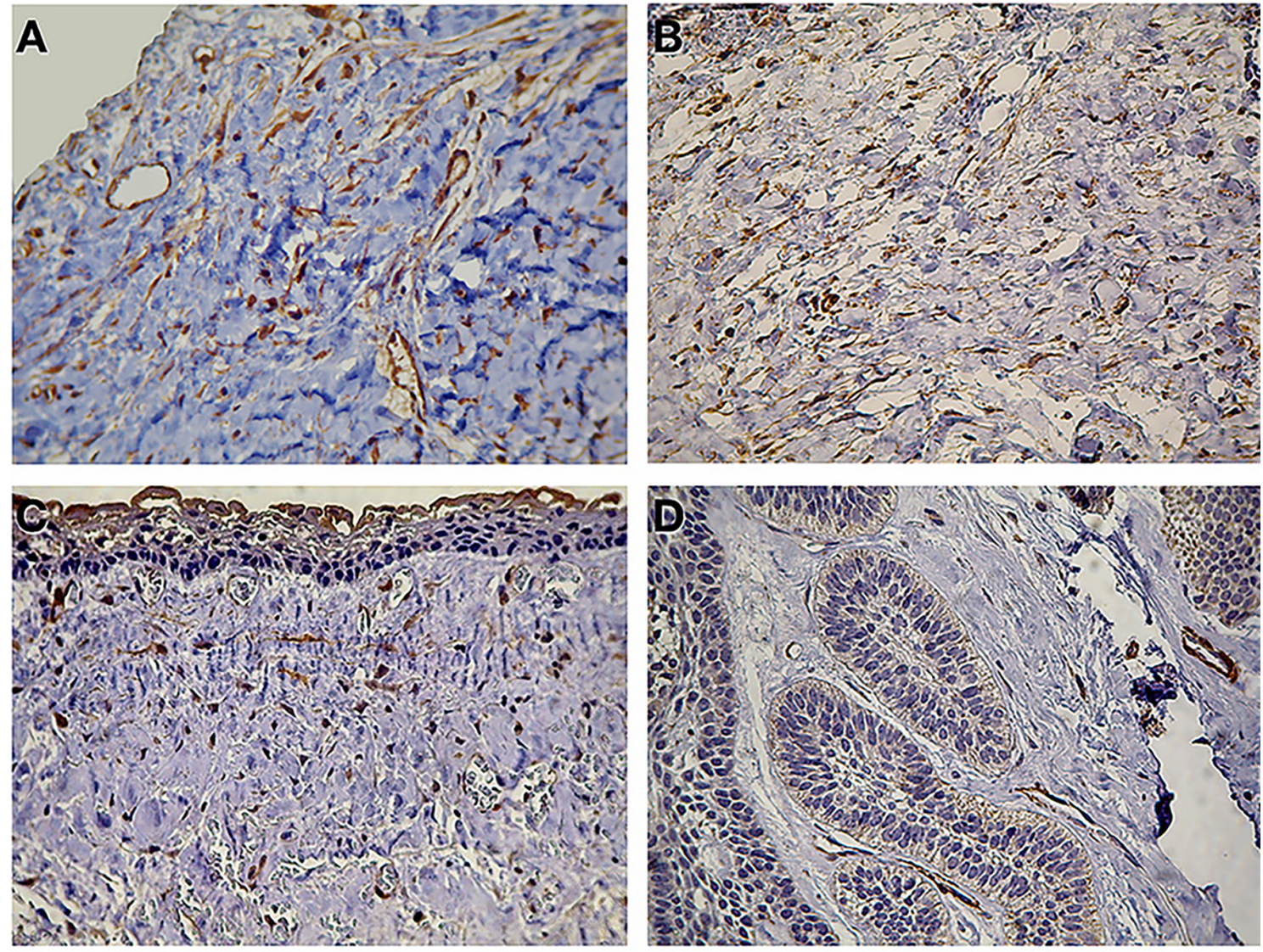

Figura 2. Fotomicrografia da expressão de $\alpha$-SMA caracterizada pela coloração marrom difusa do citoplasma (LSAB, $\times 400$ ): (A) Ceratocisto odontogênico com cápsula de tecido conjuntivo fibroso; (B) Folículo

Pericoronário com tecido conjuntivo fibroso e ausência de inflamação; (C) Ameloblastoma unicístico com epitélio de revestimento tipicamente ameloblástico suportado por uma cápsula de tecido conjuntivo fibroso;

(D) Ameloblastoma com ilhas de epitélio odontogênico circundadas por tecido conjuntivo fibroso.

Ao analisar e comparar a expressão entre os grupos, a expressão de $\alpha$-SMA e Ki-67 foi estatisticamente maior em $\mathrm{CO}$, em relação à expressão de ameloblastomas (AM+AU) e FP (Tabela 2). Por outro lado, ao comparar a expressão destes marcadores entre subtipos de ameloblastomas, não foi observada diferença estatística (Tabela 2).

0 teste de Spearman revelou uma correlação positiva $(r=0,381)$ entre a expressão de Ki-67 no epitélio e $\alpha$-SMA no tecido conjuntivo das amostras.

\section{DISCUSSÃO}

O comportamento biológico do CO é reconhecidamente mais agressivo do que o de outros cistos de desenvolvimento. Por essa razão, nos últimos anos, a discussão sobre a classificação do CO como tumor ou cisto manteve-se ativa, refletindo a preocupação em relação às características clínicas peculiares desta lesão, como elevada recorrência, potencial infiltrativo e presença de mutações gênicas ${ }^{1,2}$. Diante disso, o estudo da etiopatogênese desta lesão por meio de marcadores proteicos e moleculares é de extrema importância para o melhor entendimento do seu comportamento biológico.

Neste estudo, a expressão de Ki-67 foi maior em casos de CO, em relação à expressão observada nos ameloblastomas e FP, demonstrando o alto potencial proliferativo do epitélio cístico desta lesão. Estes mesmos resultados foram observados em estudos semelhantes ${ }^{13,15}$. A alta expressão de Ki-67 em CO poderia estar associada ao potencial de crescimento e invasão 
observado nesta patologia, uma vez que a expressão de Ki-67 já foi associada com o comportamento clínico e o potencial de recorrência de outras lesões ${ }^{9}$. Por outro lado, outros fatores, além do potencial proliferativo, também poderiam estar associados ao comportamento clínico do CO, como a remoção incompleta da lesão ${ }^{16}$. Diferente de outros cistos, o CO possui uma cápsula e um revestimento epitelial cístico friável, os quais podem facilmente ser fragmentados durante a remoção cirúrgica. Tal característica, somada à presença de cistos satélites na cápsula e o potencial proliferativo do epitélio, poderia contribuir para a alta taxa de recorrência observada nestas lesões ${ }^{16}$.

Ao analisar a expressão de Ki-67 em AM e AU, não foi observada diferença estatística entre eles, o que está de acordo com outros estudos ${ }^{17}$. Em contrapartida, Meer et al. ${ }^{18}$, ao compararem a expressão de Ki-67 entre os subtipos de ameloblastomas, encontraram uma maior expressão em $\mathrm{AU}$, concluindo que não há relação entre a taxa de proliferação epitelial e a diferença no comportamento clínico entre os subtipos de ameloblastomas. Os autores afirmaram ainda que a morfologia complexa do subtipo AM muitas vezes dificulta sua remoção completa, o que poderia estar associado com sua alta taxa de recorrência ${ }^{18}$. Dessa maneira, seria possível que a alta taxa de recorrência observada em AM esteja relacionada à remoção incompleta da lesão, em vez da expressão de Ki-6718. Entretanto, existem estudos que encontraram uma associação do potencial de infiltração e a taxa de recorrências destas lesões com o alto índice proliferativo e à elevada expressão de Ki-67 observada nas células tumorais ${ }^{19}$. Estes achados conflitantes poderiam ser atribuídos às diferenças nas metodologias utilizadas para avaliar a atividade proliferativa nesses estudos, reforçando a necessidade do uso de métodos padronizados que permitam a reprodutibilidade e a comparação dos dados. Frente a isso, dados publicados a respeito do potencial proliferativo do epitélio neoplásico dos subtipos de AM ainda são controversos.

A ativação de MF no estroma das lesões também deve ser considerada no potencial de crescimento e desenvolvimento das mesmas ${ }^{12}$. Neste estudo, a presença de MF foi avaliada pela expressão de $\alpha$-SMA e esta foi maior em casos de CO, em relação a AM e FP. Estes achados estão de acordo com outros estudos semelhantes ${ }^{10}$. Por outro lado, no estudo de Syamala et al. ${ }^{20} \mathrm{e}$ Santos et al. ${ }^{21}$, a expressão deste marcador foi maior em AM em relação a CO, e segundo Annegowda et al.12, não houve diferença na expressão de $\alpha$-SMA entre ameloblastomas e CO. Em relação à expressão semelhante de $\alpha$-SMA observada entre ameloblastomas e FP, os resultados diferem de estudos anteriores ${ }^{13,14}$. No estudo de Dutra et al. ${ }^{13}$, a expressão de $\alpha$-SMA foi maior em AM em relação à expressão de FP. Por outro lado, segundo Ramos et al. ${ }^{14}$, a expressão deste marcador foi maior em FP em relação à expressão em CO. A presença de MF em FP, sendo esta semelhante ou não à das lesões em estudo, poderia ser justificada pelo remodelamento ósseo que ocorre durante a erupção dentária ${ }^{22}$.

A presença de MFs poderia estar associada ao comportamento agressivo de cistos dentígeros, CO e ameloblastomas, conforme sugerido por estudos anteriores ${ }^{11}$. Assim, ainda que os resultados da literatura sejam controversos em relação à expressão de $\alpha$-SMA em lesões odontogênicas, faz-se necessário ressaltar que o crescimento de neoplasias está ligado a um estroma que dê condições para tal ${ }^{23}$. A interação entre MFs e células epiteliais poderia causar alterações no estroma destas lesões, o que favoreceria o crescimento, a progressão e, até mesmo, o prognóstico das mesmas ${ }^{24}$. Os MFs são considerados células importantes no remodelamento do tecido conjuntivo, uma vez que interagem com as células epiteliais, sintetizam enzimas capazes de degradar a matriz extracelular e são capazes de controlar a progressão, a invasão e a angiogênese tumoral ${ }^{24}$. No presente estudo, uma possível interação entre o potencial proliferativo do epitélio e a presença de MFs no tecido conjuntivo pode ser observada na correlação positiva entre a expressão de Ki-67 e $\alpha$-SMA. 


\section{CONCLUSÃO}

Os resultados deste estudo mostraram uma elevada expressão de Ki-67 e $\alpha$-SMA em CO, e isto poderia estar associado ao comportamento mais agressivo deste cisto em relação aos outros cistos de desenvolvimento. A expressão semelhante de Ki-67 entre AM e AU poderia sugerir que outros fatores, além do potencial proliferativo, são responsáveis pela diferença no comportamento clínico destas lesões. Possivelmente, a interação entre o índice de proliferação epitelial e a presença de MFs, observada na correlação positiva entre Ki-67 e $\alpha$-SMA, poderia ser uma das possíveis vias envolvidas na progressão de lesões como CO e ameloblastomas; entretanto, esta interação e sua relação com o comportamento clínico destas lesões precisam ser confirmadas com estudos futuros.

\section{AGRADECIMENTOS}

Este trabalho foi parcialmente financiado pela Coordenação de Aperfeiçoamento de Pessoal de Nível Superior - Brasil (CAPES) - Código financeiro 001.

\section{REFERÊNCIAS}

1. Barnes L, Eveson JW, Reichart P, Sidransky D. Neoplasms and tumour-like lesions arising from the odontogenic apparatus and maxillofacial skeleton: introduction. In: Barnes L, Eveson JW, Reichart PA, Sidransky D, editors. World Health Organization Classification of tumours pathology and genetics of head and neck tumours. 3rd ed. Lyon: IARC Press; 2005. p. 285-6.

2. Wright JM, Vered M. Update from the 4th Edition of the World Health Organization Classification of Head and Neck Tumours: odontogenic and maxillofacial bone tumors. Head Neck Pathol. 2017 Mar;11(1):68-77. http://dx.doi.org/10.1007/s12105-017-0794-1. PMid:28247226.

3. Silva YS, Stoelinga PJW, Naclerio-Homem MDG. Recurrence of nonsyndromic odontogenic keratocyst after marsupialization and delayed enucleation vs. enucleation alone: a systematic review and metaanalysis. Oral Maxillofac Surg. 2019 Mar;23(1):1-11. http://dx.doi.org/10.1007/s10006-018-0737-3. PMid:30498866.

4. Menon S. Keratocystic odontogenic tumours: etiology, pathogenesis and treatment revisited. J Maxillofac Oral Surg. 2015 Sep;14(3):541-7. http://dx.doi.org/10.1007/s12663-014-0734-5. PMid:26225042.

5. Hendra FN, Van Cann EM, Helder MN, Ruslin M, de Visscher JG, Forouzanfar T, et al. Global incidence and profile of ameloblastoma: a systematic review and meta-analysis. Oral Dis. 2019. In press. http://dx.doi.org/10.1111/odi.13031. PMid:30614154.

6. Fregnani ER, Perez DEC, Almeida OP, Kowalski LP, Soares FA, Alves FA. Clinicopathological study and treatment outcomes of 121 cases of ameloblastomas. Int J Oral Maxillofac Surg. 2010 Feb;39(2):145-9. http://dx.doi.org/10.1016/j.ijom.2009.11.022. PMid:20045283.

7. Heikinheimo K, Jee KJ, Niini T, Aalto Y, Happonen RP, Leivo I, et al. Gene expression profiling of ameloblastoma and human tooth germ by means of a cDNA microarray. J Dent Res. 2002 Aug;81(8):525-30. http://dx.doi.org/10.1177/154405910208100805. PMid:12147741.

8. Hall RC, Embery G. The use of immunohistochemistry in understanding the structure and function of the extracellular matrix of dental tissues. Adv Dent Res. 1997 Nov;11(4):478-86. http://dx.doi.org/10.1177/08959374970110041601. PMid:9470508.

9. Abdel-Aziz A, Amin MM. EGFR, CD10 and proliferation marker Ki67 expression in ameloblastoma: Possible role in local recurrence. Diagn Pathol. 2012 Feb;7(1):14. http://dx.doi.org/10.1186/17461596-7-14. PMid:22300665. 
10. Modi TG, Chalishazar M, Kumar M. Expression of Ki-67 in odontogenic cysts: a comparative study between odontogenic keratocysts, radicular cysts and dentigerous cysts. J Oral Maxillofac Pathol. 2018 Jan-Apr;22(1):146. http://dx.doi.org/10.4103/jomfp.JOMFP_94_16. PMid:29731577.

11. Nadalin MR, Fregnani ER, Silva-Sousa YT, Perez DEC. Presence of myofibroblasts and matrix metalloproteinase 2 in adicular cysts, dentigerous cysts, and keratocystic odontogenic tumors: a comparative immunohistochemical study. J Endod. 2012 Oct;38(10):1363-7. http://dx.doi.org/10.1016/j.joen.2012.05.020. PMid:22980178.

12. Annegowda VM, Devi HU, Rao K, Smitha T, Sheethal HS, Smitha A. Immunohistochemical study of alpha-smooth muscle actin in odontogenic cysts and tumors. J Oral Maxillofac Pathol. 2018 MayAug;22(2):188-92. http://dx.doi.org/10.4103/jomfp.JOMFP_31_18. PMid:30158770.

13. Dutra KL, Cordeiro MM, Vieira DS, Rivero ER. Immunohistochemical expression of matrix metalloproteinases in ameloblastomas and pericoronal follicles. J Oral Pathol Med. 2016 Sep;45(8):586-90. http://dx.doi.org/10.1111/jop.12411. PMid:26694521.

14. Ramos GO, Costa A, Meurer MI, Vieira DS, Rivero ER. Immunohistochemical analysis of matrix metalloproteinases (1, 2, and 9), Ki-67, and myofibroblasts in keratocystic odontogenic tumors and pericoronal follicles. J Oral Pathol Med. 2014 Apr;43(4):282-8. http://dx.doi.org/10.1111/jop.12131. PMid:24822270.

15. Brito-Mendoza L, Bologna-Molina R, Irigoyen-Camacho ME, Martinez G, Sánchez-Romero C, MosquedaTaylor A. A comparison of Ki67, Syndecan-1 (CD138), and molecular RANK, RANKL, and OPG triad expression in odontogenic keratocyts, unicystic ameloblastoma, and dentigerous cysts. Dis Markers. 2018 Jul;2018:7048531. http://dx.doi.org/10.1155/2018/7048531. PMid:30151060.

16. Coşarcă AS, Mocan SL, Păcurar M, Fülöp E, Ormenişan A. The evaluation of Ki67, p53, MCM3 and PCNA immunoexpressions at the level of the dental follicle of impacted teeth, dentigerous cysts and keratocystic odontogenic tumors. Rom J Morphol Embryol. 2016;57(2):407-12. PMid:27516012.

17. Bologna-Molina R, Mosqueda-Taylor A, Molina-Frechero N, Mori-Estevez AD, Sánchez-Acuña G. Comparison of the value of PCNA and Ki-67 as markers of cell proliferation in ameloblastic tumors. Med Oral Patol Oral Cir Bucal. 2013 Mar;18(2):e174-9. http://dx.doi.org/10.4317/medoral.18573. PMid:23229269.

18. Meer S, Galpin JS, Altini M, Coleman H, Ali H. Proliferating cell nuclear antigen and Ki67 immunoreactivity in ameloblastomas. Oral Surg Oral Med Oral Pathol Oral Radiol Endod. 2003 Feb;95(2):213-21. http://dx.doi.org/10.1067/moe.2003.62. PMid:12582363.

19. Ahlem B, Wided A, Amani L, Nadia Z, Amira A, Faten F. Study of Ki67 and CD10 expression as predictive factors of recurrence of ameloblastoma. Eur Ann Otorhinolaryngol Head Neck Dis. 2015 Nov;132(5):275-9. http://dx.doi.org/10.1016/j.anorl.2015.08.016. PMid:26386615.

20. Syamala D, Suresh R, Janardhanan M, Savithri V, Anand PP, Jose A. Immunohistochemical evaluation of myofibroblasts in odontogenic cysts and tumors: a comparative study. J Oral Maxillofac Pathol. 2016 May-Aug;20(2):208-13. http://dx.doi.org/10.4103/0973-029X.185898. PMid:27601810.

21. Santos PPA, Nonaka CFW, Freitas RA, Pereira Pinto L, Souza LB. Immunohistochemical analysis of myofibroblasts, TGF- $\beta 1$, and IFN- $\gamma$ in epithelial odontogenic lesions. J Oral Pathol Med. 2017 May;46(5):365-70. http://dx.doi.org/10.1111/jop.12494. PMid:27627864.

22. Hosoya A, Nakamura H, Ninomiya T, Yoshiba K, Yoshiba N, Nakaya H, et al. Immunohistochemical localization of $\alpha$-smooth muscle actin during rat molar tooth development. J Histochem Cytochem. 2006 Dec;54(12):1371-8. http://dx.doi.org/10.1369/jhc.6A6980.2006. PMid:16924123.

23. Gadbail AR, Mankar Gadbail MP, Hande A, Chaudhary MS, Gondivkar SM, Korde S, et al. Tumor angiogenesis: role in locally aggressive biological behavior of ameloblastoma and keratocystic odontogenic tumor. Head Neck. 2013 Mar;35(3):329-34. http://dx.doi.org/10.1002/hed.22960. PMid:22431258. 
24. Desmoulière A, Guyot C, Gabbiani G. The stroma reaction myofibroblast: a key player in the control of tumor cell behavior. Int J Dev Biol. 2004;48(5-6):509-17. http://dx.doi.org/10.1387/ijdb.041802ad. PMid:15349825.

\section{CONFLITOS DE INTERESSE}

Os autores declaram não haver conflitos de interesse.

\section{*AUTOR PARA CORRESPONDÊNCIA}

Kamile Leonardi Dutra-Horstmann, UFSC - Universidade Federal de Santa Catarina, Centro de Ciências da Saúde, Departamento de Odontologia, Campus Universitário, Trindade, Caixa Postal 476, 88040-900 Florianópolis - SC, Brasil, e-mail: kamileldutra@gmail.com

Recebido: Julho 2, 2019

Aprovado: Setembro 30, 2019 